\title{
Study on structure, electrical and dielectric properties of pure and Sr-doped LaCoO3 for chemical sensor application.
}

\author{
N Alhaqbani ${ }^{1}$, S Almotairi ${ }^{1}$ \\ ${ }^{1}$ Department of Chemistry, College of Science, King Saud University, Riyadh 11451, Saudi Arabia \\ nhokbany@ksu.edu.sa
}

A perovskite $\mathrm{LaCoO} 3$ was prepared by combustion and citrate-gel methodsin order to test its possible application as a chemical sensor. The crystal evolution, structure, composition, texture, morphology, and particle size were analyzed by X-ray diffraction, BET and scanning electron microscopies. The effects of doped $\mathrm{Sr}+2$ on crystal structure distortion, magnetic, and electrical properties with perovskites structure $\mathrm{La} 1 \mathrm{xSrxCoO} 3$ where $(\mathrm{x}=0.2$, $0.5,0.8$ ) have been investigated also. It was found that $\mathrm{La}+3$ is gradually replaced by $\mathrm{Sr}+2$, the changes from monoclinic to high symmetry cubic with the structure $\mathrm{La} 0.2 \mathrm{Sr} 0.8 \mathrm{CoO} 3$, resulting in a change in the bond angle as well as the bond length between Co-O. The changes in bond angle and bond length influence both electrical and magnetic properties of the system as expected. Crystal spilt Calculations predict that the bigger size of $\mathrm{Sr}+2$ reducing the splitting energy gap thus improving the electronic conductivity of perovskites. Also, $\mathrm{Sr}+2$ doping converts $\mathrm{Co}+3$ to $\mathrm{Co}+4$ and brings holes in the p-type semiconductor. Thus, increasing the resistance variation range of $\mathrm{LaCoO} 3$, enhancing the sensing performance. The temperature variation of the resistivity shows that these compounds have semiconductor behavior. The frequency dependence of the dielectric constant in these materials indicates that space charge polarization contributes significantly to their observed dielectric parameters. Keywords: perovskite LaCoO3, Rare earth, chemical sensors, dielectric properties, electrical resistivity.
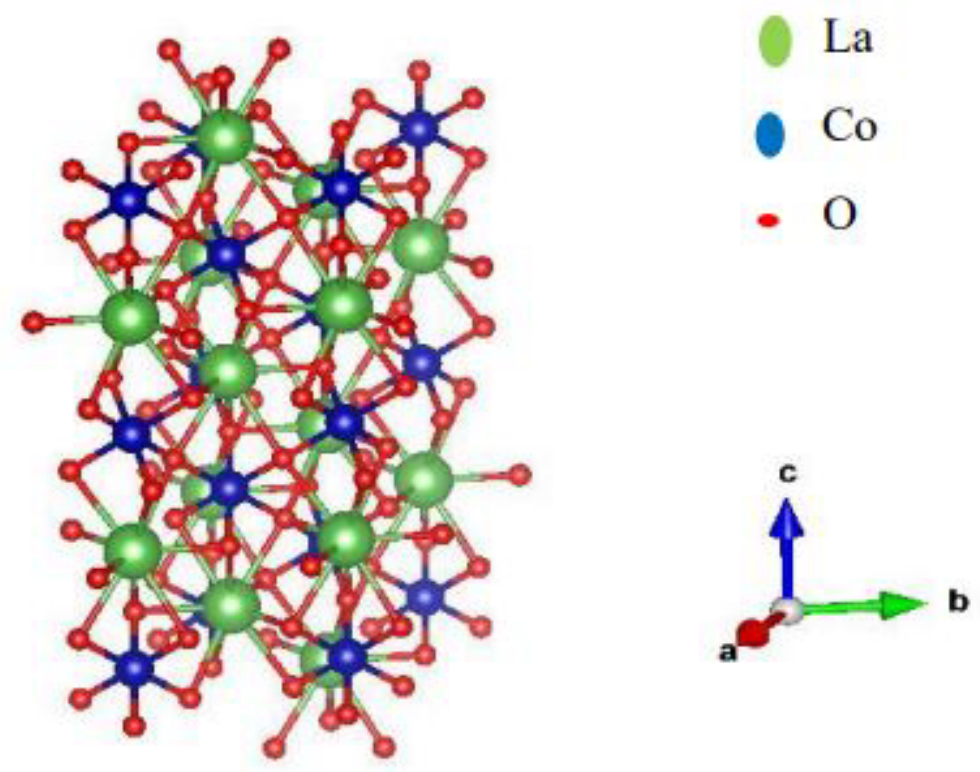

Figure 1

Acta Cryst. (2020). A76, a78 